\title{
A further family with congenital renal proximal tubular dysgenesis
}

\author{
Paul MacMahon, Ronald A S Blackie, Michael J House, Rupert A Risdon, Martin d'A Crawfurd
}

\begin{abstract}
A new syndrome of oligohydramnios, Potter's syndrome, and anuric renal failure leading to stillbirth or neonatal death from respiratory failure has recently been described. Histologically, there is renal tubular dysgenesis, especially of the proximal tubules, and apparent glomerular crowding. To date, five families have been reported, in four of which there have been affected sibs and in two parental consanguinity. The disorder is, therefore, thought to be inherited in an autosomal recessive manner.
\end{abstract}

Allanson et $a l^{1}$ reported a family in which two successive girls were stillborn after premature births associated with oligohydramnios and Potter's syndrome. Renal histology in both cases showed absence of normal proximal tubules. The second child was also microcephalic, had a congenital heart defect,

Department of Paediatrics, Charing Cross Hospital, London W6 8RF.

P MacMahon*

Department of Histopathology, Charing Cross and Westminster Medical School, London W6 8RF.

R A S Blackiet

Department of Obstetrics, West London Hospital, London W6 7DQ.

M J House

Department of Histopathology, The Hospital for Sick Children, Great Ormond Street, London WCIN 3JH.

R A Risdon

Kennedy Galton Centre, Clinical Research Centre, Northwick Park Hospital, Watford Road, Harrow, Middlesex HA1 3UJ.

M d'A Crawfurd

Correspondence to Dr Crawfurd.

*Present address: Paediatric Unit, Waterford Regional Hospital, Ardkeen, Waterford, Ireland.

†Present address: J S Pathology Services Ltd, 81 Harley Street, London W1N 1DE.

Received for publication 30 June 1989.

Accepted for publication 21 November 1989. and multiple joint contractures. These two infants differed in some respects from the subsequently reported cases (table), and may possibly represent a different disorder of proximal renal tubules.

Voland $e t a l^{2}$ reported two families, both with parental consanguinity. In the first family a boy was affected and in the second family three boys were affected. As in the family of Allanson et $a l^{1}$ the kidneys were characterised by proximal tubular dysgenesis and the glomeruli appeared crowded together.

The first detailed description of this disorder was given by Schwartz et al. ${ }^{3}$ They reported a case in which a woman had severe oligohydramnios in two successive pregnancies. Both pregnancies ended in the birth of an infant, one male and the other female, who died within 24 hours of birth with an anuric acidosis and pulmonary hypoplasia. Apart from joint contractures and a fractured femur in the first infant there were no other abnormalities. The parents were not consanguineous. The mother gave a history of moderate alcohol intake and cigarette smoking in the first pregnancy, and had had an overdose of cocaine at 25 weeks in the second. In both infants the gross appearance of the kidneys was unremarkable with normal cortical surfaces, corticomedullary differentiation, and pelvicalyceal systems. Histologically, the kidneys of both infants showed tubular immaturity with some increase in interstitial fibrous tissue. The immature tubules had hyperchromatic nuclei, prominent nucleoli, scant cytoplasm, and were all negative to periodic acid-Schiff reagent. Immunoperoxidase staining with epithelial membrane antigen failed to show the normal negative staining of proximal tubules. The tubular immaturity resulted in a relative crowding of normal looking glomeruli.

The authors postulated autosomal recessive inheritance, vascular impairment, possibly owing to cocaine, although maternal cocaine was only documented for the second pregnancy, or a defect in a maternal growth factor as possible aetiologies. They discussed earlier reports of tubular hypoplasia, in one of which there was also an arrest of glomerular maturation, ${ }^{5}$ the other being that of Allanson et al ${ }^{1}$ mentioned above. They considered that neither of these reports fully corresponded to the findings in their own cases. The mother in their family has since 
given birth to a third normal child (Schwartz, personal communication).

Most recently Swinford $e t a l^{4}$ reported a further family in which they described two affected brothers in detail and gave a history of two earlier affected brothers. As in the family of Schwartz et al ${ }^{3}$ there was oligohydramnios, Potter's syndrome, and anuria, followed by death shortly after birth. The renal abnormality was similarly characterised by absence of normal proximal tubules, confirmed by negative PAS staining of the brush borders, and positive immunoperoxidase staining of primitive proximal tubules. There was also both a relative and an absolute increase in numbers of normal glomeruli. They introduced the term tubular dysgenesis for this new renal disorder.
We present a female infant born to unrelated parents with a picture very similar to that described by Schwartz et $a l^{3}$ and by Swinford $e t a l .{ }^{4}$ This is the first report of renal tubular dysgenesis from the United Kingdom or indeed from Europe.

\section{Case report}

A 29 year old woman, gravida 2 para 0 , with a previous missed abortion in April 1986, delivered a female infant at 39 weeks' gestation in March 1987. The birth weight was $2830 \mathrm{~g}$. The mother had been found to have severe oligohydramnios on ultrasound scanning at 18 and 20 weeks of pregnancy. Subsequently no liquor at all could be detected and the

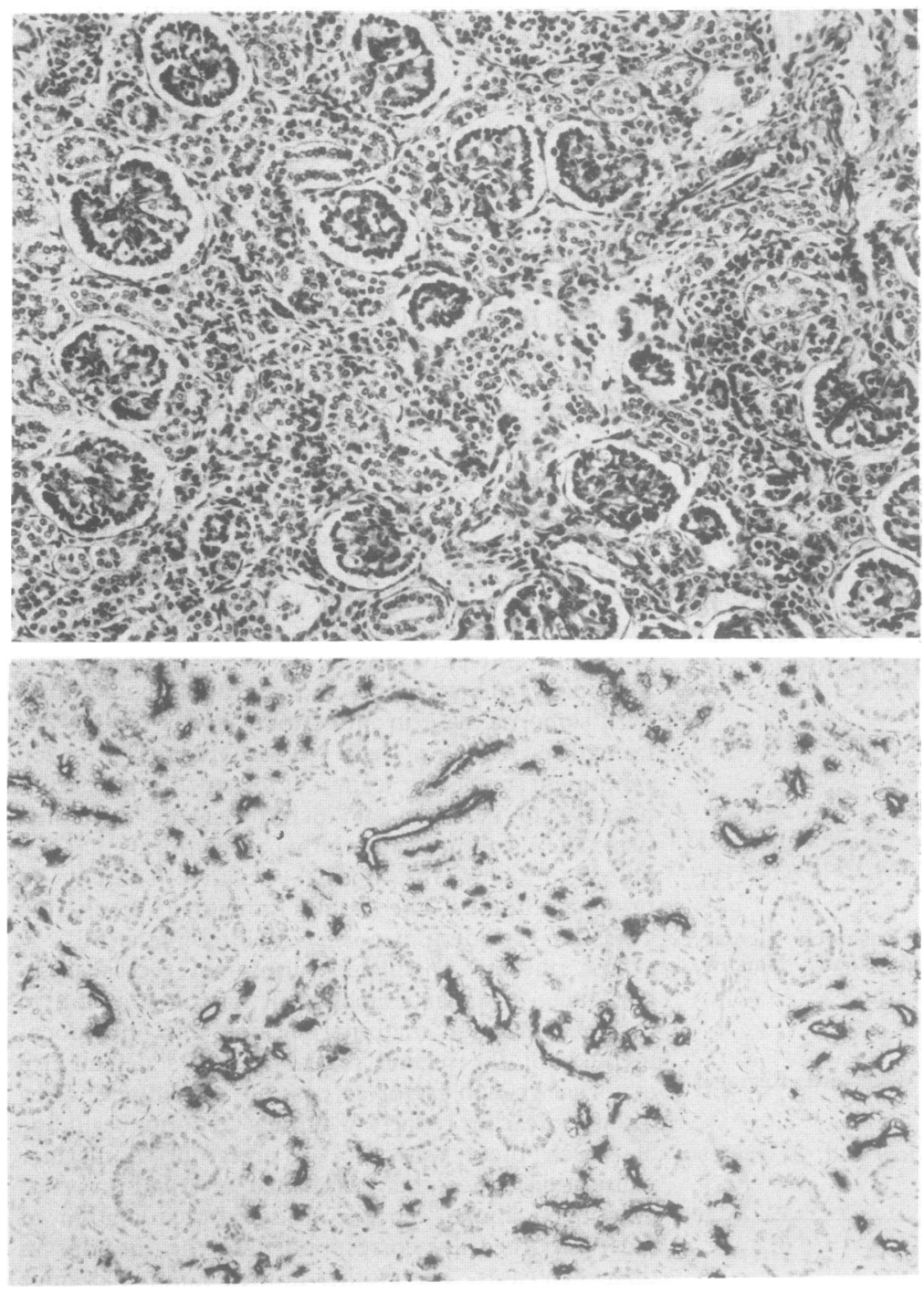

Figure 1 Renal histology showing tubular dysgenesis and glomerular crowding.

Figure 2 Immunostaining of kidney with epithelial membrane antigen showing positive staining of all tubules and therefore lack of mature proximal tubules. 


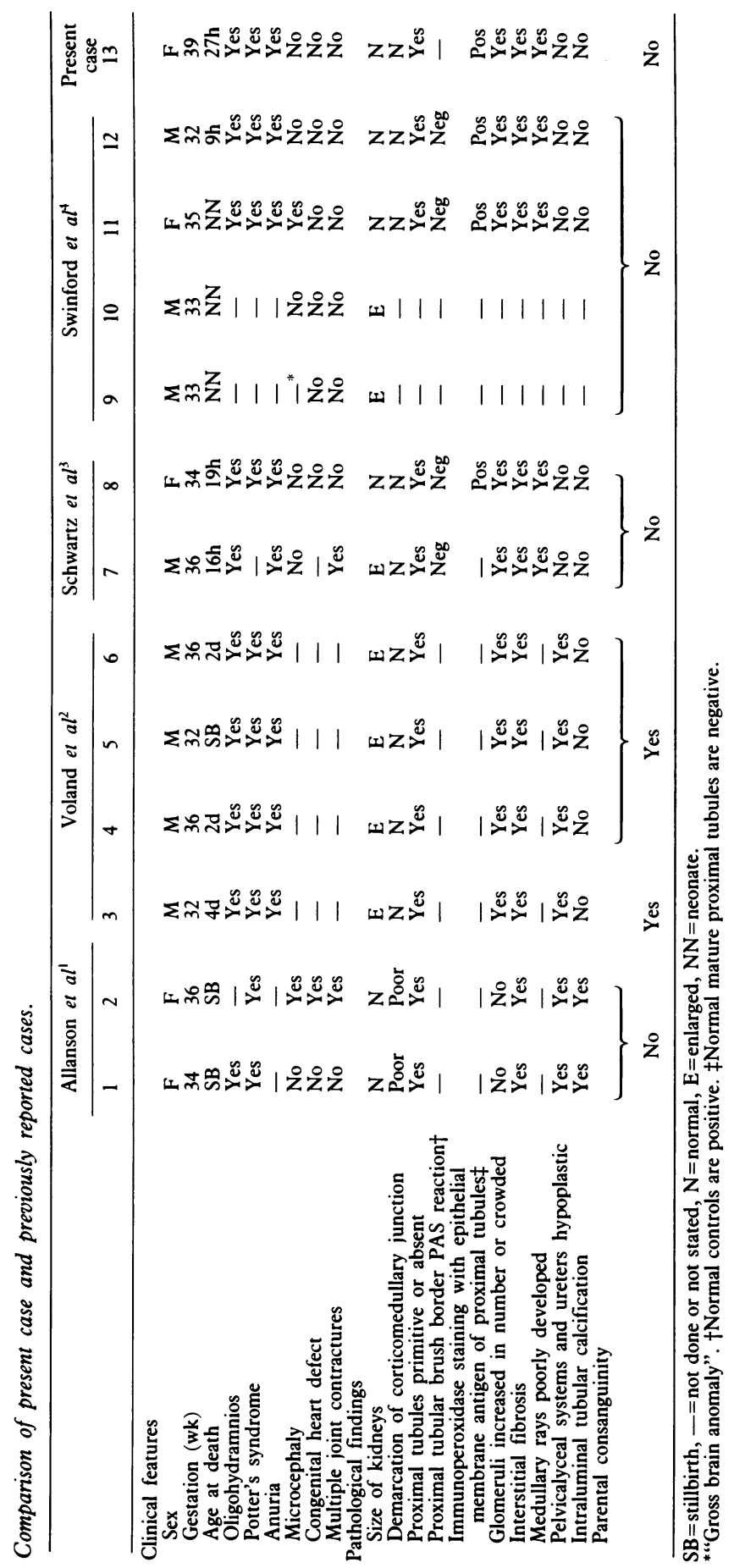


fetus showed marked deformation. Further ultrasound examination nevertheless showed normal sized fetal kidneys. Fetal chromosomes showed no abnormality.

The mother was well apart from Gilbert's syndrome, from which her brother also suffers. She is unrelated to her 30 year old husband, who is well.

Because of a marginal reduction in fetal growth she was induced at 39 weeks resulting in a normal vaginal delivery. The infant had severe deformation of the head, chest, and hips consistent with Potter's syndrome, and suffered severe respiratory difficulties. She was completely anuric throughout life and died at 27 hours despite appropriate treatment in an intensive care unit.

The parents were seen three months later for genetic counselling and were advised that recurrence risks were probably either 1 in 4 or very low. They were seen again seven months after the birth when one of us had seen the paper by Schwartz et $a l^{3}$ and a revised risk of almost 1 in 4 explained. They have subsequently suffered a further miscarriage at 12 weeks, but have a normal son born on 8.11.88.

\section{NECROPSY FINDINGS \\ Gross findings}

There was Potter's facies with petechial haemorrhages over the forehead. The lungs were haemorrhagic and congested but were aerated. There were no gross abnormalities in the cardiovascular, gastrointestinal, endocrine, reticuloendothelial, or central nervous systems. The kidneys showed normal fetal lobulation. The renal cortices were slightly pale, but the kidneys and the rest of the genitourinary system appeared otherwise grossly normal.

\section{Histology of the kidneys}

All the proximal tubules were small with reduced thickness of lining epithelial cells and small cross sectional areas. In places there was a slight excess of interstitial connective tissue and the glomeruli appeared relatively crowded together. The cortex was slightly thinned and the medulla also a little attenuated. There was no evidence of maldifferentiation (fig 1). Immunostaining with epithelial membrane antigen was positive in all the tubules confirming an absence of mature proximal tubules (fig 2).

\section{Discussion}

Like the previously described infants the infant reported here shows tubular, especially proximal tubular, hypoplasia or dysgenesis with a slight increase in interstitial fibrosis, but no evidence of renal dysplasia. The fact that all the tubules gave a positive immunoperoxidase stain with epithelial membrane antigen, as in the cases of Schwartz et al ${ }^{3}$ and Swinford $e t a l,{ }^{4}$ confirms the absence of normally developed proximal tubules.

The clinical course and histological appearances in this infant are almost identical to those in the earlier reports ${ }^{34}$ and she must be assumed to have the same disorder (table).

There was no history of maternal illness, drugs, or irradiation in the present case which, taken together with the sib occurrence and parental consanguinity in the previous reports, strongly favours an autosomal recessive aetiology for this new syndrome. In view of the history of the two spontaneous abortions in this family, and that of Allanson et al, ${ }^{1}$ it cannot be excluded that these may in some way be related to the genetic defect.

The patient reported by Lorentz and Trillo ${ }^{5}$ with glomerular as well as tubular immaturity probably represents a different disorder, as suggested by Schwartz et al. ${ }^{3}$ The stillborn girls reported by Allanson et $a l^{1}$ had the same basic defect of tubular dysgenesis but also had other features, both renal and extrarenal. However, both microcephaly and joint contractures have been reported individually in later cases. It is not possible to decide retrospectively whether or not their patients had the same disorder.

We are grateful to Mrs Sheila Kingsley for typing the manuscript.

1 Allanson JE, Pantzar JT, MacLeod PM. Possible new autosomal recessive syndrome with unusual renal histopathological changes. Am $\mathcal{F}$ Med Genet 1983;16:57-60.

2 Voland JR, Hawkins EP, Wells TR, Saunders B, Jones M, Benirschke K. Congenital hypernephronic nephromegaly with tubular dysgenesis: a distinctive inherited renal anomaly. Pediatr Pathol 1985;4:231-45.

3 Schwartz BR, Lage JM, Pober ER, Driscoll SG. Isolated congenital renal tubular immaturity in siblings. Hum Pathol 1986;17:1259-63.

4 Swinford AE, Bernstein J, Toriello HV, Higgins JV. Renal tubular dysgenesis: delayed onset of oligohydramnios. $A m \mathcal{J}$ Med Genet 1989;32:127-32.

5 Lorentz WB, Trillo AA. Neonatal renal failure and glomerular immaturity. Clin Nephrol 1983;19:154-9. 